University of Nebraska - Lincoln

DigitalCommons@University of Nebraska - Lincoln

U.S. Environmental Protection Agency Papers

U.S. Environmental Protection Agency

2004

\title{
Monitored Natural Attenuation Forum: A Panel Discussion
}

\author{
Bob Norris \\ Norris Environmental Consulting \\ Lenny Siegel \\ Center for Public Environmental Oversight \\ Stephen Lester \\ Center for Health, Environment and Justice \\ Hal White \\ U.S. Environmental Protection Agency
}

Follow this and additional works at: https://digitalcommons.unl.edu/usepapapers

Norris, Bob; Siegel, Lenny; Lester, Stephen; and White, Hal, "Monitored Natural Attenuation Forum: A Panel Discussion" (2004). U.S. Environmental Protection Agency Papers. 148.

https://digitalcommons.unl.edu/usepapapers/148

This Article is brought to you for free and open access by the U.S. Environmental Protection Agency at DigitalCommons@University of Nebraska - Lincoln. It has been accepted for inclusion in U.S. Environmental Protection Agency Papers by an authorized administrator of DigitalCommons@University of Nebraska - Lincoln. 


\section{Monitored Natural Attenuation Forum: A Panel Discussion}

\section{Bob Norris}

Lenny Siegel

Stephen Lester

Hal White
In the spring 2004 issue of Remediation, we presented the question that appears below to three of our MNA panel members. As discussed in the previous issue, "MNA should be used very cautiously as the sole remedy" (Fran Kremer, U.S. Environmental Protection Agency [US EPA]). It should be the lowest importance compared to other natural attenuation processes and "public involvement is particularly important in cases where dilution plays a substantial role in the remediation strategy" (Barbara Bekins, U.S. Geological Survey). "Definitions and evaluation strategies must explicitly address what processes are acting and the footprints of those processes. Failing to address them is a flaw that justifies scientific and public concern" (Bruce Rittmann, Northwestern University). We would like to expand on these ideas in this issue of the journal, specifically the issue of protecting health versus meeting regulatory requirements, the risks (being open and honest with the public) associated with natural attenuation, and the role dilution plays with other active remedies. We received additional responses on this topic from four more panel members and have included them here.

Question: In its report on natural attenuation, the NRC's Committee on Intrinsic Remediation convened a panel of representatives from communities located near contaminated sites to discuss "Community Concerns About Natural Attenuation." One of the principal concerns of the panel was that current natural attenuation policy "legitimizes the dilution and dispersion of toxic chemicals."The panel of community activists "strongly urged the NRC to define natural attenuation as a process of degradation and / or transformation that excludes dilution and dispersion processes."Are these concerns merely a public perception issue, or do they highlight a more fundamental flaw in how sites are addressed through natural attenuation remedies? Should dilution be excluded in natural attenuation remedy evaluations either as a contributing mechanism or as a sole mechanism?

\section{Panel Member: Bob Norris, Director, Norris Environmental Consulting, Longmont, $\mathrm{CO}$}

\section{CONSIDERATIONS WHEN EVALUATING DILUTION AS PART OF MNA}

The intent here is not to answer questions regarding the role of dilution in MNA but to raise some issues to consider within some broader contexts when evaluating MNA.

The first response by many to the concept of dilution as the sole solution or as a contribution to the solution to impacted groundwater is not generally positive. However, it is important to look at dilution from several different perspectives. Furthermore, it is the responsibility of the professional(s) working on the site to conduct a thorough technical 
.. reliance on dilution places a heavy burden on the professionals proposing to incorporate dilution as a significant contributor to attenuation. review, come to appropriate and defensible conclusions, and finally present the results and conclusions in a way that addresses all stakeholders' concerns.

Before identifying dilution as a site-specific issue, it is necessary to consider whether it could play a significant role in the decision-making process. If groundwater is highly impacted, the contribution from dilution is likely to be minimal. The contribution from other attenuation mechanisms (preferably destructive mechanisms) will dwarf the contribution from dilution and, thus, relying solely on dilution will not be an issue. Where dilution is the primary attenuation mechanism, it is likely not to be significant.

Therefore, MNA is not appropriate. For highly impacted sites, dilution will only play a role at the margins of the plume where its impact will be greatest when infiltration is significant or the plume pinches out before entering a broader flow regime. Thus, dilution might impact the extent of the plume to a relatively minor degree, and the question then becomes whether dilution can be relied on where the far extent of the plume approaches or extends past a point of compliance. Dilution within highly impacted plumes will not play a role in achieving plume-wide MCLs (maximum contaminant levels) or similar remediation goals in a time frame consistent with other remedies.

In order for dilution to be an important mechanism, concentrations of the constituents of concern (COCs) will only marginally exceed groundwater standards and, thus, the risk to begin with will be minimal. Unless utilized drinking water supplies are impacted or imminently threatened, there may actually be more risk related to mobilizing to the site and conducting drilling and other activities than is presented by the impacted groundwater. (This, of course, ignores state regulations concerning deterioration of state waters.)

In either case, the extent to which dilution contributes to attenuation will generally be difficult to document given the normal variability in groundwater data. Thus, reliance on dilution places a heavy burden on the professionals proposing to incorporate dilution as a significant contributor to attenuation.

Where uncertainty remains, which is almost every site for which MNA or another remedy is selected, risk can be managed by appropriate locations of sentinel wells, sampling protocols, and contingency plans. Where uncertainty in the MNA analysis is above average, the long-term monitoring requirements should be more robust whether dilution is or is not a significant mechanism.

In way of a broader perspective, there are many situations under which dilution has been an acceptable method of mitigating risk. Examples include where groundwater discharges into a fast-moving surfacewater body (stream) such that a very substantial dilution occurs within a very short distance. At many sites, obtaining a permit to discharge to a POTW (publicly owned treatment works) is based on demonstration that the COCs will be sufficiently diluted. Another example of dilution is smokestack emissions and effluents from vapor recovery (soil vapor extraction) where average wind velocities, height of discharge, and distance from property lines are used to calculate permissible discharges. In all cases, there may be a perception of a decision being made on economical grounds even when all regulatory considerations are being met.

The most important reason for addressing impacted groundwater is to manage risk to human health and the environment. Risk evaluation is not an exact science and, thus, as with most of what we do, judgment is required in order to achieve appropriate solutions for environmental concerns. An example of where judgment is required is the federal regulations for feasibility/corrective measure studies at Comprehensive Environmental Response, Compensation and Liability Act (CERCLA) and Resource 
Conservation and Recovery Act (RCRA) sites. CERCLA provides for a number of Applicable or Relevant and Appropriate Requirements (ARARs), including short-term and long-term risks, as well as balancing considerations, one of which is cost.

Another perspective relates to what the risks are of selecting MNA with dilution compared to other potential exposures to chemicals of concern. For example, chlorinated drinking water, especially where the water source is surfacewater, is allowed to contain up to $100 \mu \mathrm{g} / \mathrm{L}$ of trihalomethanes. Trihalomethanes is a surrogate measurement for the large number of halogenated compounds that result from chlorinating water containing natural organics such as humates. Almost certainly, we have addressed and continue to address groundwater with lower concentrations of less toxic compounds than we typically consume in our drinking water. This does not imply that we should not be concerned about the additional impact from groundwater, only that our judgment on final outcomes should be based on a breadth of considerations to allow the public to have a better perspective of how decisions regarding MNA and other site cleanup approaches compare to other risks.

Finally, we should be aware of potentially changing cleanup requirements. For example, indoor air impacts resulting from vapor-phase transport of dissolved-phase constituents in groundwater is a topic of concern for US EPA and many state agencies. At some sites, this can lead to lower cleanup targets than currently exist. Dissolvedphase VOCs (volatile organic compounds) are in equilibrium with the vadose-phase gas (air or oxygen-depleted air). Through diffusion or air movement enhanced by partial vacuums within buildings, VOCs can enter basements or first floors of buildings. As the VOCs move through the unsaturated soils, attenuation mechanisms, including adsorption, degradation, and dilution, may take place. The process of evaluating the risks from indoor air intrusion has similarities to how MNA of groundwater is evaluated. The use of models such as the Johnson and Ettinger model is currently a controversial topic. The role of such models in MNA procedures or protocols is likely to be a topic of discussion over the next few years. It is likely that the contribution of dilution to attenuation will be controversial and face the same issues and perceptions.

\section{Guest Panel Member: Lenny Siegel, Director, Center for Public Environmental Oversight, Mountain View, CA}

Informed members of the public recognize that "monitored natural attenuation" is not necessarily a do-nothing remedy. In fact, community activists often consider destructive forms of natural attenuation, such as intrinsic bioremediation, preferable to remedies that simply shift contamination from one medium to another or one location to another.

However, in many cases, responsible parties and regulators propose natural attenuation as a remedy without fully considering, or at least analyzing, to what degree actual degradation is taking place. This is not so much rooted in policy - most natural attenuation guidance documents emphasize the importance of degradation - as it is in practice in the field. It is reinforced by the definitions of natural attenuation, which typically include various forms of dilution, unacceptable as a remedy to most environmental activists.

The slogan "Dilution is not the solution to pollution" is not simply a remnant of past debate but a continuing challenge. In fact, dilution is the principal response, at the point of service, to the world's largest toxic plume, the poisoning of the Colorado River with perchlorate from the Kerr-McGee rocket-fuel plant in Las Vegas Wash. Tens of millions of
Informed members of the public recognize that "monitored natural attenuation" is not necessarily a do-nothing remedy. 
... we learned, from activists from throughout the United States, that trust is the key to public stakeholder consideration of natural attenuation. people downstream now drink perchlorate-laced water, and untold more ingest lettuce and other agricultural products containing perchlorate from Colorado River irrigation water.

Operators of the largest California water district supplying Colorado River water are able to keep the perchlorate concentration in tap water below official action levels by blending it with water unpolluted with perchlorate from northern California. I, along with other environmental advocates, question whether serving half as much perchlorate to twice as many people achieves any public health benefit. If health protection, as opposed to meeting regulatory targets, were the objective, water treatment systems would be installed.

By definition, natural attenuation takes place whether or not it is recognized as an official environmental response. The question is: To what degree should it be relied upon to reach environmental objectives? In general, my colleagues and I are much more willing to accept the role dilution plays after the application of active remedies. That is, if pump-and-treat or more modern forms of treatment are employed, and they lose their effectiveness over time, we may be willing to let diffusion, dispersion, and advection, as well as natural degradation, "polish off" residual contamination.

We recognize that the continuing operation of costly cleanup systems, once they have reached a point of diminishing returns, does little to protect public health and the environment. But the answer to the asymptotic effect observed at a multitude of groundwater sites is not simply to declare "natural attenuation" and go home. Rather, it calls for "adaptive site management," a reevaluation of remedies based upon the slope of the concentration-over-time curve, not the absolute level of the asymptote.

That is, once the original remedy has lost its effectiveness, decision makers should consider not only terminating the remedy, but also optimizing the use of the original technology or evaluating other technologies for bringing contamination levels down. They should also consider the potential role that continuing research and development might play in completing the cleanup task. Dilution processes might play a role, but they should be analyzed against a full suite of remedy adjustments. This adaptive site management approach was, in fact, the principal recommendation of another NRC committee, the Committee on Navy Remediation, which issued its report, "Environmental Cleanup at Navy Facilities: Adaptive Site Management," in early 2003. I was a member of that committee.

However, most of the people who are impacted by toxic pollution do not judge proposed environmental responses, such as a reliance on natural attenuation, by reviewing detailed technology descriptions or studying the graphs described by the NRC Navy Committee. They ask, instead, can we trust the polluters and regulators at this site to look out for our interests, or are they simply trying to cut costs and walk away - the traditional "wink and wave" approach?

In late summer 1998, my organization (the Center for Public Environmental Oversight) convened the National Stakeholders' Forum on Monitored Natural Attenuation, bringing together nearly 250 scientists, activists, and government officials to consider the suitability of natural attenuation in environmental cleanup. We organized a series of scientific presentations and expected intense technical debate.

Instead, we learned, from activists from throughout the United States, that trust is the key to public stakeholder consideration of natural attenuation. If the public believes that those responsible for cleanup decisions are just trying to evade responsibility, it will reject any form of natural attenuation, even if the PowerPoint presentation promises permanent degradation. 
On the other hand, when the public believes that responsible parties and officials are both honest and willing to shape their decisions to meet public concerns, the public appears willing to evaluate the lines of evidence for natural attenuation, or any other remedy, on their merits.

\section{Panel Member: Stephen Lester, Science Director, Center for Health, Environment and Justice, Falls Church, VA}

Community members affected by contaminated sites have generally not accepted the selection of natural attenuation as an option to clean up contaminated groundwater. There are fundamentally two reasons why people have difficulty accepting this remedial option. The primary reason is the lack of documentation that genuine natural degradation processes, rather than dilution and dispersion, are predominantly responsible for the reduction in observed contaminant levels. This issue is at the heart of the public's reluctance to support natural attenuation as a remedy for cleaning up contaminated groundwater.

The second reason is that many community members view dilution and dispersion mechanisms as ways to let industry off the hook for the contamination they've caused by allowing the contamination to slowly spread into the environment instead of cleaning it up. Including dilution and dispersion mechanisms as natural attenuation processes has significantly contributed to why many community members consider natural attenuation to be a "do-nothing" approach to cleanup. With natural attenuation, there's no active remediation, no on-site treatment that reduces risks. Instead, contamination is left in place for long periods of time to take its own course. In contrast, most community members want the contamination cleaned up as quickly as possible and object to remedies that call for leaving significant levels of contamination on site.

Remedies that provide no on-site treatment are rejected by community members not only because they feel that their health may be jeopardized by the lack of active cleanup, but also because they see it as fundamentally wrong to let a company off the hook for polluting the environment. Grassroots community activists have long held several fundamental beliefs about the cleanup of contaminated sites: (1) if you make a mess, you need to clean it up; (2) moving waste from one place (or medium) to another is not cleanup; and (3) a company should be held accountable for the pollution it generates. The dilution and dispersion of contaminants goes against the very premise of these principles.

Furthermore, dilution and dispersion mechanisms do not destroy contaminants or alter their inherent toxicity, and including them as natural attenuation processes not only legitimizes these mechanisms as options for addressing contaminated groundwater, but it also equates them to genuine degradation processes such as biological and chemical degradation and physical immobilization that essentially destroy or alter the inherent toxicity of contaminants. Mechanisms that are nondestructive and allow contaminants to spread into the environment without altering their inherent toxicity should not be equated to mechanisms that destroy a contaminant or alter its inherent toxicity.

Including dilution and dispersion as natural attenuation processes also creates confusion about what mechanisms are really responsible for reducing contaminant levels in groundwater. Several key questions need to be answered to eliminate this confusion: 1) How much do each of the different attenuation processes contribute to the overall reduction in contaminant levels measured in groundwater? 2) How much of a reduction is the result of dispersion, dilution, evaporation, and other nondestructive
With natural attenuation, there's no active remediation, no on-site treatment that reduces risks. 
Community members want to be assured that genuine natural degradation processes are responsible for most of the reduction in observed contaminant levels. processes and how much is the result of genuine degradation and/or transformation processes? and 3) How long will it take for genuine degradation processes to effectively reduce contaminant levels?

Community members want to be assured that genuine natural degradation processes are responsible for most of the reduction in observed contaminant levels. They recognize that there are ways to identify that genuine degradation processes are occurring. However, this is not enough. They also need to be able to correlate the degree of degradation to the reduction in contaminant levels in groundwater.

Without this documentation, there's no way to distinguish between dilution and dispersion mechanisms and genuine degradation mechanisms or to evaluate the effectiveness of the individual attenuation processes. As a result, community members are likely to attribute contaminant reduction to dilution and dispersion and other nondestructive mechanisms and view decisions to use natural attenuation as being driven more by its low cost than by scientific evidence.

The community panel convened by the NRC Committee described the need for this documentation as the "public's greatest concern." It remains so today. The challenge now is for regulators and companies to devote the time and resources necessary to provide the evidence needed to document the degree to which genuine degradation processes are responsible for contaminant losses in groundwater. This will help convince the public that genuine degradation processes, not dilution or dispersion, are predominantly responsible for the contaminant losses observed in groundwater.

This lack of documentation remains a fundamental flaw in evaluating the effectiveness of natural attenuation. This is not a matter of public perception but a matter of the public wanting to see hard scientific evidence that defines what processes are responsible for contaminant losses.

For these reasons, dilution, dispersion, and other nondestructive processes should be considered separately from genuine degradation processes and not included in definitions of natural attenuation. As long as dispersion and dilution are included as natural attenuation processes, communities will continue to object to this option as a legitimate cleanup alternative. Until clear documentation is provided that shows people how genuine degradation processes, and not dilution and dispersion, are responsible for the reduction in the concentration of contaminants in groundwater, this alternative will remain unacceptable to most community members.

\section{Guest Panel Member: Hal White, U.S. Environmental Protection Agency, Office of Solid Waste and Emergency Response, Washington, DC}

The finding by the NRC Committee that US EPA's "natural attenuation policy 'legitimizes the dilution and dispersion of toxic chemicals" misinterprets Agency policy (specifically, OSWER Directive 9200.4-17P, entitled "Use of Monitored Natural Attenuation at Superfund, RCRA Corrective Action, and Underground Storage Tank Sites"). First, the Directive distinguishes between "natural attenuation processes" and the reliance on these processes as a remediation alternative defined as "monitored natural attenuation." Monitoring at sites that rely on natural attenuation processes to achieve remediation objectives within a reasonable time frame is critical — without monitoring, there is no remediation approach. Stated another way, natural attenuation by itself (without adequate monitoring) is not acceptable as a remediation alternative. Second, the 
Directive merely lists all the processes that comprise "natural attenuation" without regard to which may be applicable to any particular contaminant (or suite of contaminants) at any particular site, because omitting any process would yield an incomplete and inaccurate definition. Simply ignoring or denying the existence of processes one doesn't like has no effect on their actual existence. Third, the Directive is very carefully worded to make it clear that "those processes that degrade or destroy contaminants" (as opposed to processes that do not, such as dilution and dispersion) are "preferred" for monitored natural attenuation remedies. The Directive also makes it clear monitored natural attenuation "will only be appropriate for sites that have a low potential for contaminant migration," which is essentially what dilution and dispersion are all about. However, the fact remains that processes such as dilution and dispersion do occur whether or not they are "preferred."

Bob Norris is director of Norris Consulting in Longmont, Colorado. He has developed and implemented bioremediation technologies for 20 years, taught several courses on monitored natural attenuation, and managed the monitored natural attenuation aspect of several remediation projects. He currently serves as an advisory board member for Remediation and is a panel chair for this MNA forum.

Lenny Siegel has been Executive Director of the Center for Public Environmental Oversight (formerly CAREER/PRO) since 1994. He has been director of the Pacific Studies Center, in Mountain View, since 1970. He is one of the environmental movement's leading experts on military facility contamination, and he has served on numerous advisory committees in that area. His organization runs Internet forums both on military environmental issues and brownfields.

Stephen Lester has been the science director for the Center for Health, Environment and Justice since 1981. He got his start in working with communities affected by toxic waste sites when he was hired by the New York State Department of Health to be the technical advisor to the residents of Love Canal in Niagara Falls, New York. His primary work involves translating technical jargon into everyday language for communitybased organizations and helping people understand the role of science and technical information in local community struggles. He also directs the Center's research efforts, which have produced over 100 guidebooks and fact-packs. Mr. Lester has sat on numerous scientific advisory and has participated in several peer review committees. Mr. Lester has a master's degree in toxicology from the Harvard University School of Public Health and a master's degree in environmental health from the New York University Institute of Environmental Medicine.

Hal White is employed as an environmental scientist and has been with the EPA's Office of Underground Storage Tanks since 1994. He chaired the Agency workgroup that developed the Monitored Natural Attenuation Directive (D9200.4-17P). His forum contribution is intended to clarify certain points in the Directive and not to establish Agency policy. The Directive is the official source for EPA policy regarding monitored natural attenuation. 


\section{PANEL MEMBERS}

Dr. Robert C. Borden

North Carolina State University

Raleigh, NC

Dr. Frank H. Chapelle

USGS

Columbia, SC

Mr. Austin I. Cooley

Brown and Caldwell

Houston, TX

Dr. David E. Ellis

Dupont Company

Wilmington, DE

Mr. Mark Ferrey

Minnesota Pollution Control Agency

St. Paul, MN

Dr. Fran Kremer

USEPA/Office of

Research and Development

Cincinnati, $\mathrm{OH}$

Dr. Michael D. Lee

Terra Systems

Wilmington, DE

Mr. Stephen Lester

Center for Health,

Environment \& Justice

Falls Church, VA

Dr. Michelle Lorah

USGS

Baltimore, MD

Mr. Ken Lovelace

USEPA

Washington, DC
Dr. Perry McCarty

Stanford University

Stanford, CA

Mr. Ross Miller

Parsons

South Jordan, UT

Dr. Charles J. Newell

Groundwater Services, Inc.

Houston, TX

Dr. Robert D. Norris

Norris Environmental Consulting

Longmont, $\mathrm{CO}$

Dr. Evan K. Nyer

ARCADIS G \& M, Inc.

Tampa, FL

Dr. Hanadi S. Rifai

University of Houston

Houston, TX

Dr. Bruce E. Rittmann

Northwestern University

Evanston, IL

Dr. Guy Sewell

East Central University

Ada, OK

Dr. C. Herbert Ward

Rice University

Houston, TX

Mr. Todd Wiedemeier

T.H. Wiedemeier \& Associates, LLC

Denver, CO

Dr. John T. Wilson

USEPA/RS Kerr Laboratory

Ada, OK 